\title{
Evaluation of model-based methods in estimating respiratory mechanics in the presence of variable patient effort
}

 \\ ${ }^{a}$ Centre for Bioengineering, University of Canterbury, Private Bag 4800, Christchurch, New Zealand \\ ${ }^{b}$ School of Engineering, Monash University Malaysia, Jalan Lagoon Selatan, Bandar Sunway, 47500 Subang Jaya, \\ Selangor, Malaysia
}

\begin{abstract}
Monitoring of respiratory mechanics is required for guiding patient-specific mechanical ventilation settings in critical care. Many models of respiratory mechanics perform poorly in the presence of variable patient effort. Typical modelling approaches either attempt to mitigate the effect of the patient effort on the airway pressure waveforms, or attempt to capture the size and shape of the patient effort. This work analyses a range of methods to identify respiratory mechanics in volume controlled ventilation modes when there is patient effort. The models are compared using 4 Datasets, each with a sample of 30 breaths before, and 2-3 minutes after sedation has been administered to reduce patient efforts, where the underlying pulmonary mechanical properties should be unchanged over such a small period. Model identified parameters from breathing cycles with patient effort are compared to breathing cycles that do not have patient effort. All models have advantages and disadvantages, so model selection may be specific to the respiratory mechanics application. However, in general, the combined method of iterative interpolative pressure reconstruction, and stacking multiple consecutive breaths together has the best performance over the Dataset. The variability of identified elastance when there is patient effort is the lowest with this method, and there is little systematic offset in identified mechanics when sedation is administered.
\end{abstract}

\section{Introduction}

Continuous monitoring of patient-specific respiratory mechanics through model-based methods enables the optimisation of patient-specific mechanical ventilation (MV) therapy in the critical care environment [1-3]. However, the application of model-based methods are hindered by patients breathing spontaneously and/or asynchronous events during MV [4-6]. Most respiratory mechanics models are passive, and assume patients are fully sedated during controlled ventilation modes. However, during lower levels of sedation, patients may breathe on top of MV support [7-9]. These spontaneous breathing efforts cause highly variable airway pressure or flow profiles, and frequent inaccurate estimations of respiratory mechanics parameters if these passive models are used.

The presence of patient effort when the respiratory model does not account for it, results in poor model fit and poor parameter estimations [10]. Equally, there is currently no way to predict the magnitude or trajectory of these external inputs, as they are likely a function of patient condition, sedation, and ventilator settings. Hence, it is not practicable to incorporate the external inputs in a model. More complex models of respiratory mechanics that multiple compartments and non-linear effects can be more physiologically accurate. These models could be adapted to include compartments to represent patient breathing effort. However, the addition of model parameters to an already complex model is likely to cause these models to be practically non-identifiable with only clinically available ventilation data [10-12]. Hence, models cannot become too complex before they become unusable in a clinical setting.

Finally, the existing approaches to measure or to approximate the patient breathing effort tend to introduce additional invasiveness and cost, and are therefore less desirable for use in clinical decision

\footnotetext{
${ }^{*}$ Corresponding author

Email addresses: daniel.redmond@pg.canterbury.ac.nz (Daniel P. Redmond), chiew.yeong.shiong@monash.edu (Yeong Shiong Chiew), vincent.major65@gmail.com (Vincent Major), geoff.chase@canterbury.ac.nz (J. Geoffrey Chase) 
support systems $[13,14]$. In particular, oesophageal balloon catheters have been used to estimate pleural pressure [15]. Estimations of pleural pressure enable changes in pressure due to diaphragmatic activity to be separated from the pressure supplied by the ventilator, hence, allow better estimation of patientspecific respiratory mechanics. The use of an oesophageal pressure catheter requires experience and additional equipment to correctly place and inflate the balloon [15]. Use of oesophageal catheters is also invasive and uncomfortable for the patients [14]. Therefore, their use is not generally desirable in clinical practice.

The forced oscillation technique is another method to estimate the respiratory mechanics of the spontaneously breathing patients [16]. In this method, small amplitude pressure signals at varying frequencies are applied to the airway pressure, which can be used to identify frequency dependent respiratory mechanics, independent of patient breathing effort [17]. However, forced oscillation requires additional equipment to perform, and has so far had limited application in the clinical environment [18].

Hence, there is need for respiratory mechanics models and identification methods that can capture these spontaneous and/or asynchronous breathing efforts, while maintaining good respiratory mechanics estimations. Asynchronous events occur when the patient effort does not occur at the same time as the ventilator provides breathing support, and result in a perturbed airway pressure or flow profile [7, 8, 19].

In recent studies, several models were developed to capture or reduce the effects of asynchronous efforts or spontaneous breathing on the airway pressure waveform, to improve respiratory mechanics monitoring. For example, Major et al. [20] introduced a method by pooling airway pressure to reduce the asynchronous effects for better respiratory mechanics estimations. Redmond et al. [21] proposed a polynomial model to capture patient efforts during volume controlled ventilation modes. Damanhuri et al. [22] reconstructs airway pressure to minimise these asynchronous effects by building up the missing part of the pressure profile. Vicario et al. [23] proposed a constrained optimisation approach to capture patient respiratory mechanics and spontaneous breathing efforts during pressure support ventilation. All these methods provide opportunity to improve respiratory mechanics estimations during MV.

To provide consistent respiratory mechanics monitoring for mechanically ventilated patients, these models need to be capable of responding to changes in the frequency and timing of patient effort. This paper presents a comparison of these models/methods. In this study, the performance of these selected models was evaluated using MV data that contains spontaneous breathing efforts during volume controlled ventilation. This study will provide a comprehensive understanding of the problem faced during MV respiratory mechanics estimations and the advantages and disadvantages of each model/method.

\section{The Models \& Algorithms}

All of the models presented here are investigated using data from patients ventilated on volume control mode. During volume control ventilation, patient efforts/asynchrony are seen as perturbations of the independent, uncontrolled pressure profile, while the dependent flow input is controlled as expected by the ventilator. Figure 1a shows a typical airway and pressure waveform of a sedated patient and Figure $1 \mathrm{~b}$ shows the perturbations in the pressure waveform in the same patient when patient spontaneous effort occurs. Therefore, in many of these models, the airway flow and volume profiles can be considered normal, and adjustments are made to enable the use of pressure waveforms modified by patient effort.

The models presented in this comparison study take two different general approaches. The first approach is to capture, rather than remove the unmodelled patient effort, and thus model and identify the patient effort profile. These models have the additional purpose of monitoring some metric of patient effort, such as work of breathing, but do so directly as part of the model. Polynomial model of effort (see Section 2.2) and constrained optimisation (see Section 2.3) take this approach. The second approach is to remove and/or minimise the effect of the patient effort, and eliminate asynchronies. These models were developed for the purpose of respiratory mechanics estimation. Pressure reconstruction by combining breaths (see Section 2.4), and iterative pressure reconstruction (see Section 2.5) take this approach. Post-algorithm comparison of the original and reconstructed pressure waves can provide an estimate of patient effort .

\subsection{Linear single compartment model}

The linear single compartment lung model represents the airway pressure as a linear combination of flow and volume [10], as shown in Equation (1). This model assumes no patient effort and is clinically 

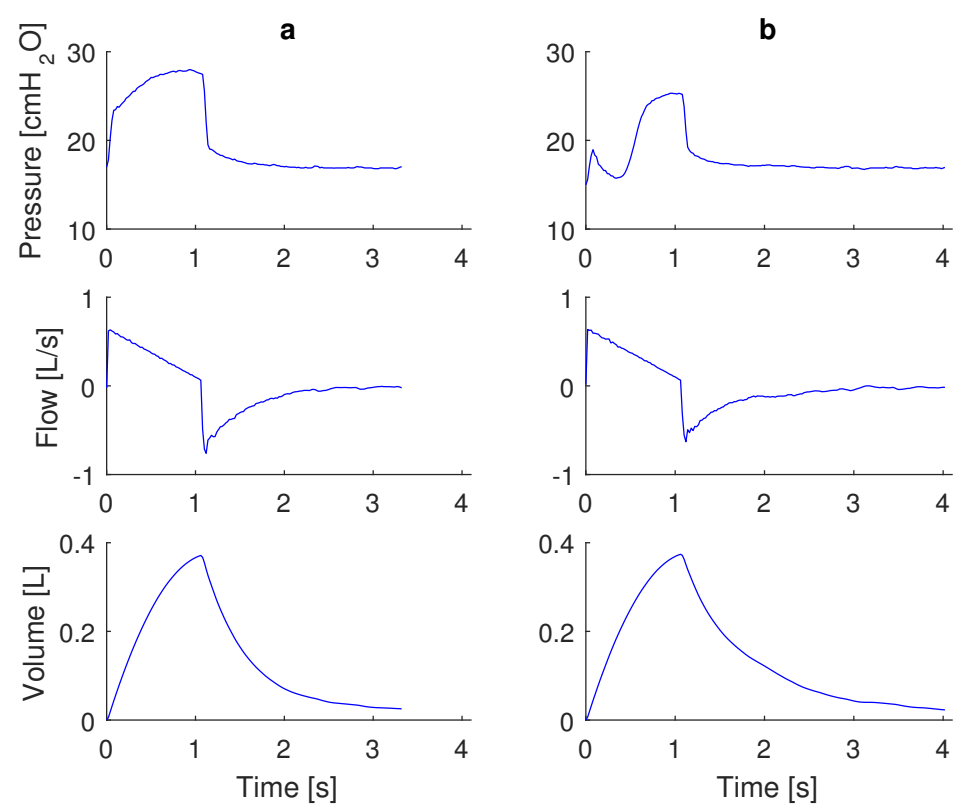

Figure 1: Sample breaths from Dataset 2. a. shows a clean pressure, flow, and volume waveform with no patient effort after sedation has been administered and b. the patient effort during a breath before sedation has been administered. As this is from volume control ventilation, the flow, and therefore volume waveforms are very similar, while the major change is in the pressure waveform.

accepted [10, 24], and will thus be used as a baseline comparison of the performance of other models when there is spontaneous breathing.

$$
P_{a w}(t)=E V(t)+R \dot{V}(t)+P_{0}
$$

In this model, $P_{a w}$ is the airway pressure, $t$ is time, $V$ is the air volume, and $\dot{V}$ is the air flow. $P_{0}$ is an offset pressure, usually positive end-expiratory pressure (PEEP) if there is no auto-PEEP [25]. $E$ is respiratory system elastance and $R$ is respiratory system resistance.

The single compartment model typically performs poorly in the presence of patient effort, and often misestimates elastance due to the anomaly in airway pressure caused by patient effort [26]. $E$ and $R$ are identified using linear least squares with measured $P_{a w}, \dot{V}$, and calculated $V$ over the inspiration of one breath [24]. Figure 2a shows how the single compartment model fits to a sample breath during inspiration with a large patient effort at the end of inspiration.

\subsection{A Polynomial model of effort}

When the patient breaths spontaneously on top of ventilator support, the diaphragm contracts, creating a negative pressure in the pleural space. This negative pressure generates a drop in the airway pressure. If the pressure drop is assumed to have a fixed shape, the magnitude and timing of this effort and the respiratory mechanics parameters can be identified [21]. In this polynomial model of effort, the patient effort, $P_{e}(t)$, is included in the single compartment model.

$$
P_{a w}(t)=E V(t)+R \dot{V}(t)+P_{0}+P_{e}(t)
$$

It is assumed to have a parabolic shape which can be described by three parameters.

$$
P_{e}(t)= \begin{cases}0 & t<t_{s} \\ a t^{2}+b t+c & t_{s} \leq t<t_{f} \\ 0 & t \geq t_{f}\end{cases}
$$

This patient effort is modelled by a quadratic function, as shown in Equation (3). In this model $t_{s}$ and $t_{f}$ represent the 'starting and finishing time' of patient effort, and are determined by the roots of the polynomial $a t^{2}+b t+c=0$. 

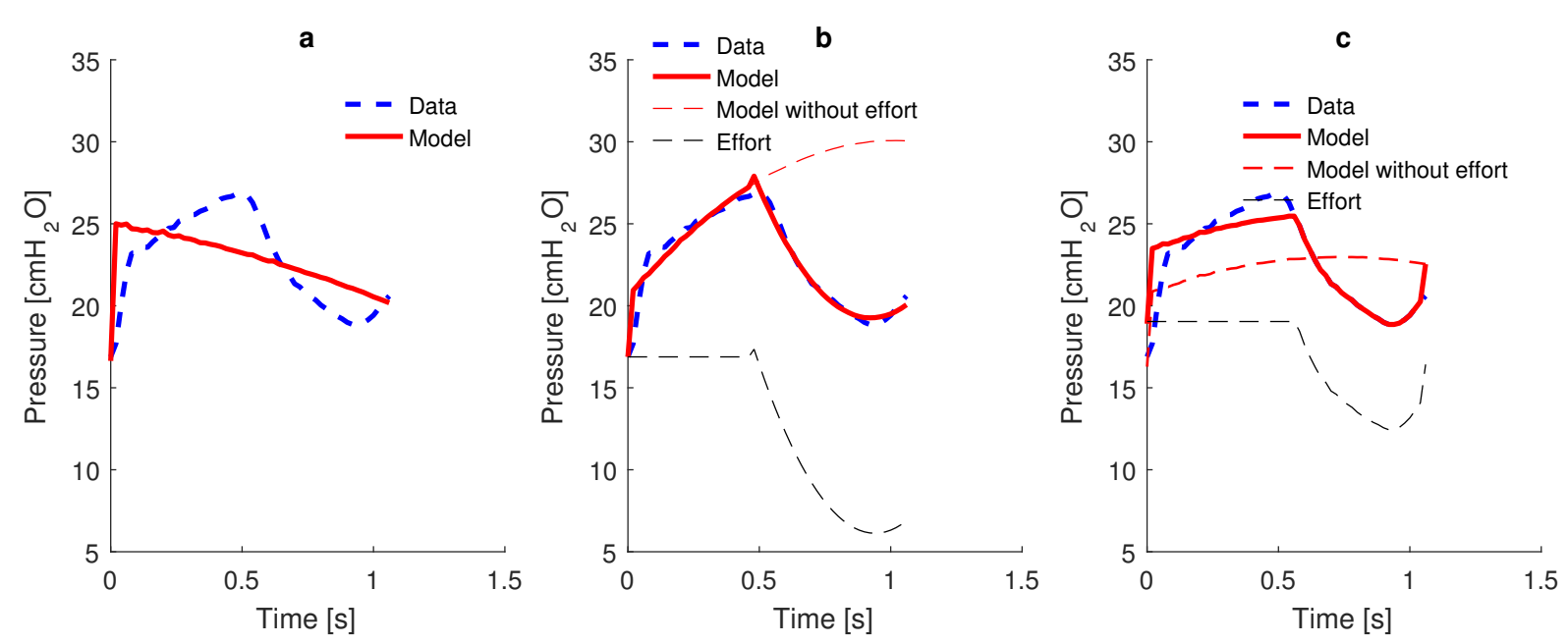

Figure 2: Model fitting to a sample breath during inspiration. a The single compartment model with identified $E=6.7$ $\mathrm{cmH}_{2} \mathrm{O} / \mathrm{L}$ and $R=13.0 \mathrm{cmH}_{2} \mathrm{Os} / \mathrm{L}$. b Polynomial effort model, showing the predicted pressure profile in the absence of patient effort. Identified $E=35.0 \mathrm{cmH}_{2} \mathrm{O} / \mathrm{L}$ and $R=6.0 \mathrm{cmH}_{2} \mathrm{Os} / \mathrm{L}$. c Constrained optimisation approach including the patient effort profile. Identified $E=9.7 \mathrm{cmH}_{2} \mathrm{O} / \mathrm{L}$ and $R=2.1 \mathrm{cmH}_{2} \mathrm{Os} / \mathrm{L}$.

An iterative multiple linear regression is used to simultaneously solve for the patient effort and respiratory mechanics parameters during inspiration [21]. Figure $2 \mathrm{~b}$ shows how the model fits to a sample breath, and the predicted pressure profile that would exist in the absence of patient effort.

\subsection{Constrained optimisation for approximating patient effort}

The constrained optimisation approach assumes certain inequality constraints on the patient pressure profile [23], rather than a fixed shape used in the polynomial model. The patient effort profile $\left(\tilde{P}_{m u s}\right)$ is allowed to monotonically decrease up to a certain point, and then monotonically increase until the end of inspiration, where it remains constant throughout expiration.

$$
\begin{array}{cl}
\tilde{P}_{\text {mus }}\left(t_{k+1}\right)-\tilde{P}_{\text {mus }}\left(t_{k}\right) \leq 0 & \text { for } k=1,2, \ldots, m-1 \\
\tilde{P}_{\text {mus }}\left(t_{k+1}\right)-\tilde{P}_{\text {mus }}\left(t_{k}\right) \geq 0 & \text { for } k=m, m+1, \ldots, q-1 \\
\tilde{P}_{\text {mus }}\left(t_{k+1}\right)-\tilde{P}_{\text {mus }}\left(t_{k}\right)=0 & \text { for } k=q, q+1, \ldots, N \\
P_{a w}(t)=E V(t)+R \dot{V}(t)+P_{0}+P_{\text {mus }}(t)
\end{array}
$$

Equations (4) shows the inequality constraints applied to $\tilde{P}_{m u s}$ which is used in place of $P_{e}$ in Equation (2), to give Equation (5). The bounds $m$ and $q$ in Equation (4) represent the time of the maximum patient effort and end of patient effort, respectively. The value of $q$ is fixed to the start of expiration. The value of $m$ is found by a search for minimum model fitting error for all value of $m$ in the range $0<m<q$. These inequality constraints, and limits on the values of $E, R$ and $\tilde{P}_{m u s}$ can be included in solving this problem by quadratic programming [23]. Figure 2c shows the fitting of this model to a sample breath, and the identified patient effort profile.

\subsection{Pressure Reconstruction by combining breaths}

Pressure reconstruction by pooling multiple breathing cycles (known as PREDATOR [27]) works on the assumption that only parts of the breath are affected by unmodelled patient effort. This assumption suggests that there are regions during inspiration where the pressure profile is "correct", or unaffected by patient effort and can be modeled by the single compartment lung model. By pooling and combining multiple breathing cycles, over up to five breaths, a larger region of the pressure waveform unaffected by patient effort can be found. The single compartment model can then be fitted only over the region of "correct" pressure during inspiration. Figure 3 illustrates the stacking process and the subsequent fitting of the single compartment model to the reconstructed pressure profile. This approach provides less variable and more accurate estimations of the true, underlying respiratory mechanics. Its disadvantage is the requirement for several breaths with unchanging ventilator settings which may not be available 



Figure 3: Pressure reconstruction by combining 5 breaths. The original breath and the additional 4 breaths are shown together with the reconstruction and the fitted model. Identified $E=25.2 \mathrm{cmH}_{2} \mathrm{O} / \mathrm{L}$ and $R=10.0 \mathrm{cmH} \mathrm{O}_{2} / \mathrm{L}$.
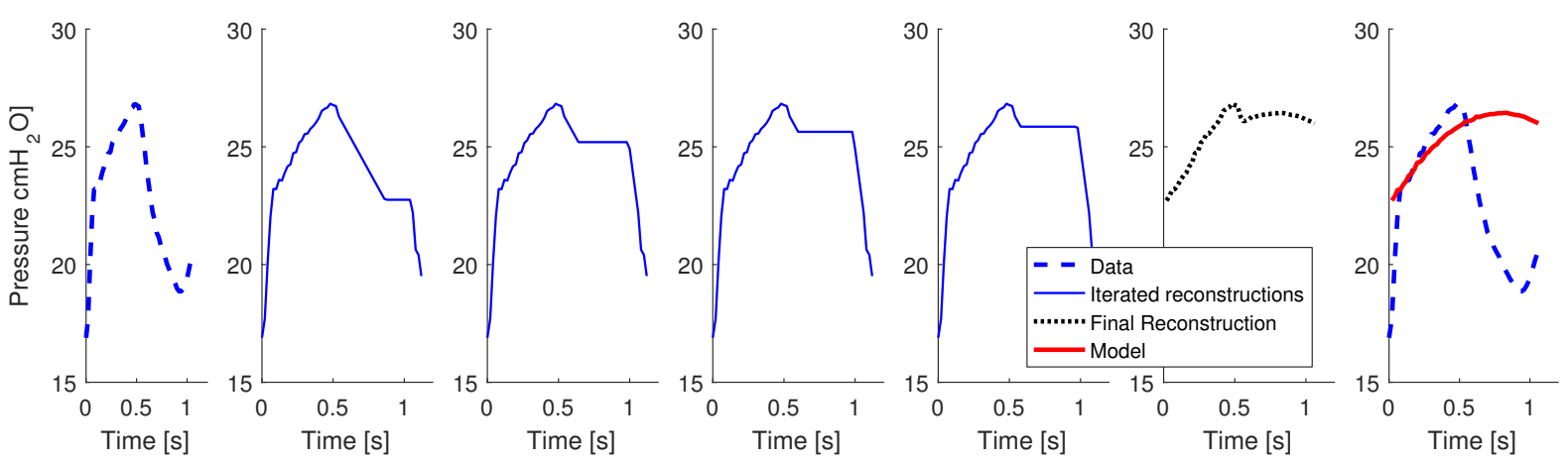

Figure 4: Iterative Pressure reconstruction performed on the sample breath. Moving from left to right shows each successive iteration, followed by the final reconstructed pressure curve and the model fitting. Identified $E=23.5 \mathrm{cmH} \mathrm{O}_{2} \mathrm{O} / \mathrm{L}$ and $R=9.2$ $\mathrm{cmH}_{2} \mathrm{Os} / \mathrm{L}$.

during major MV pressure and flow profile changes during recruitment manoeuvres. The details of this method can be found in [20,27, 28].

\subsection{Iterative interpolative pressure reconstruction}

An iterative interpolative pressure reconstruction (IIPR) method [29] identifies the presence of asynchronous events in the airway pressure profile, and reconstructs the pressure iteratively through a series of airway pressure fillings and model identification. This method is an extension of Damanhuri et al.'s simple airway pressure filling method [22] to improve the reconstruction. Once the airway pressure is iteratively reconstructed for a single inspiration, it delivers a final breath-specific elastance and resistance. This approach thus reconstructs breath by breath. Figure 4 shows the sequential reconstructions that iteratively build up the pressure profile of a volume controlled inspiration affected by asynchrony.

\subsection{Reconstructed then stacked}

In this study, the IIPR method (section 2.5) is combined with the PREDATOR method (section 2.4), and its performance in respiratory mechanics estimation is evaluated. 5 consecutive, IIPR reconstructed breaths are selected, then they are pooled, using the PREDATOR method, and the single compartment model is applied over the inspiration region. Figure 5 shows the five reconstructed breaths, and how the model fits to the maximum of the pressure waveforms.

\section{Methods}

\subsection{Data}

Four retrospective Datasets were used for this study. The patients were ventilated on synchronous intermittent mandatory ventilation mode with volume control ramp flow profile using PB-840 ventilators (Covidien-Puritan Bennet, Boulder, CO). Details of the ventilation of each patient are shown in Table 1. 

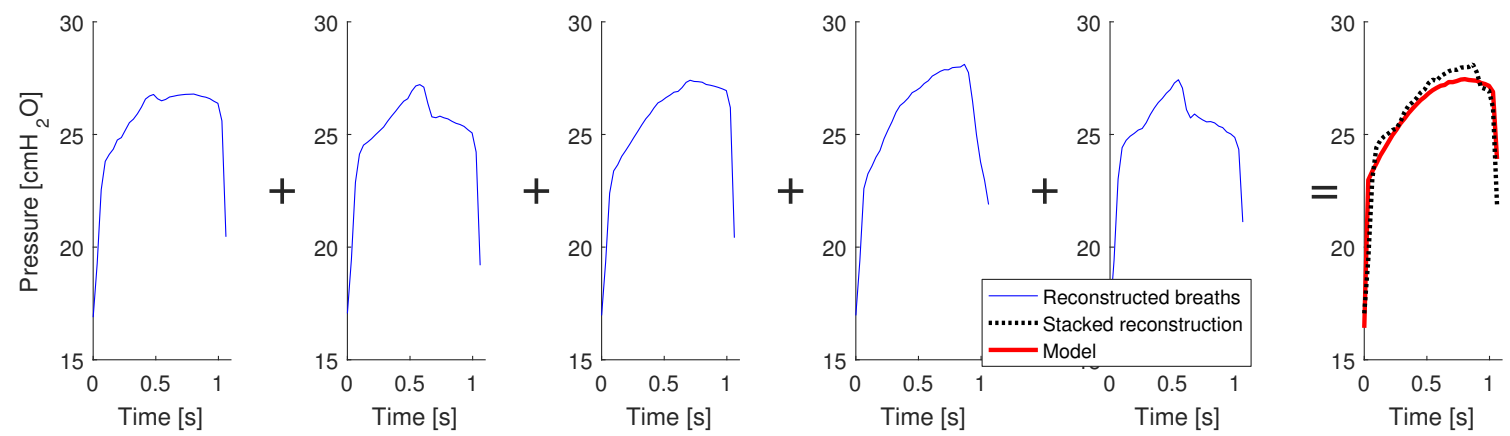

Figure 5: Stacking of five breaths that have all been iteratively reconstructed with IIPR. The five reconstructed breaths are the same as shown in Figure 3. The final stacked and reconstructed pressure profile and model fitting are shown on the right. Identified $E=26.8 \mathrm{cmH}_{2} \mathrm{O} / \mathrm{L}$ and $R=9.6 \mathrm{cmH}_{2} \mathrm{Os} / \mathrm{L}$.

Table 1: Mechanical ventilation parameters for each patient on the day the data was recorded, and the primary diagnosis for ICU admission.

\begin{tabular}{ccccc}
\hline Patient & 1 & 2 & 3 & 4 \\
\hline Primary diagnosis & Fecal Peritonitis & Fecal Peritonitis & Cardiac surgery & Pneumonia \\
Tidal Volume $[\mathrm{mL}]$ & 365 & 370 & 480 & 540 \\
Respiratory rate $[1 / \mathrm{minute}]$ & 18 & 19 & 14 & 18 \\
PEEP $\left[\mathrm{cmH}_{2} \mathrm{O}\right]$ & 15 & 17 & 11 & 13 \\
\hline
\end{tabular}

Patients were mechanically ventilated and exhibiting asynchronous spontaneous breathing on top of ventilator support. The patients were then sedated for clinical reasons. When sedation is administered, the patient's spontaneous breathing efforts cease, enabling the comparison of predicted respiratory mechanics from the pre-sedation data with the more reliable respiratory mechanics estimations from post-sedation data.

A sample of 30 consecutive breathing cycles before and approximately 3 minutes after administration of sedation was selected for this analysis. No other changes were made to mechanical ventilation between pre-sedation and post-sedation sample, or immediately prior to the pre-sedation sample. Figure 6 shows the first 10 breathing cycles from each Dataset, both pre-sedation and post-sedation. The pre-sedation figures illustrate the heterogeneous nature of the patient effort that may occur. The overall number of breaths and patients is designed to show differences in models.

\subsection{Comparison of methods}

\subsubsection{Elastance $(E)$ and Resistance $(R)$ before and after sedation}

Within a short period, and assuming no major changes occurred in patients condition as well as MV setting, it is assumed that the respiratory mechanics should exhibit only small natural variation [30]. Thus, a model should identify $E$ and $R$ values with very little variability across the 30 consecutive sampled breaths. In addition, identified $E$ and $R$ would be very similar pre- and post-sedation, possibly with a slight increase in elastance after sedation is applied [31]. The pre- and post-sedation distributions of $E$ and $R$ are then compared for each model and each Dataset. Variability, and the shift in median identified parameters are used to assess method efficiency.

\subsubsection{Variability of parameter estimations}

The variability of the parameter estimations $(E$ and $R$ ) from each model is assessed using median absolute deviation (MAD). MAD is calculated from the medians of absolute deviations from the sample median.

\section{Results}

Figures 7 and 8 show the pre-sedation and post-sedations cumulative distributions of identified elastance and resistance respectively, for each model across all Datasets. In Figure 7, it can be seen that the smallest change in median estimated elastance in Datasets 1 and 4 is with the II Predator model, 

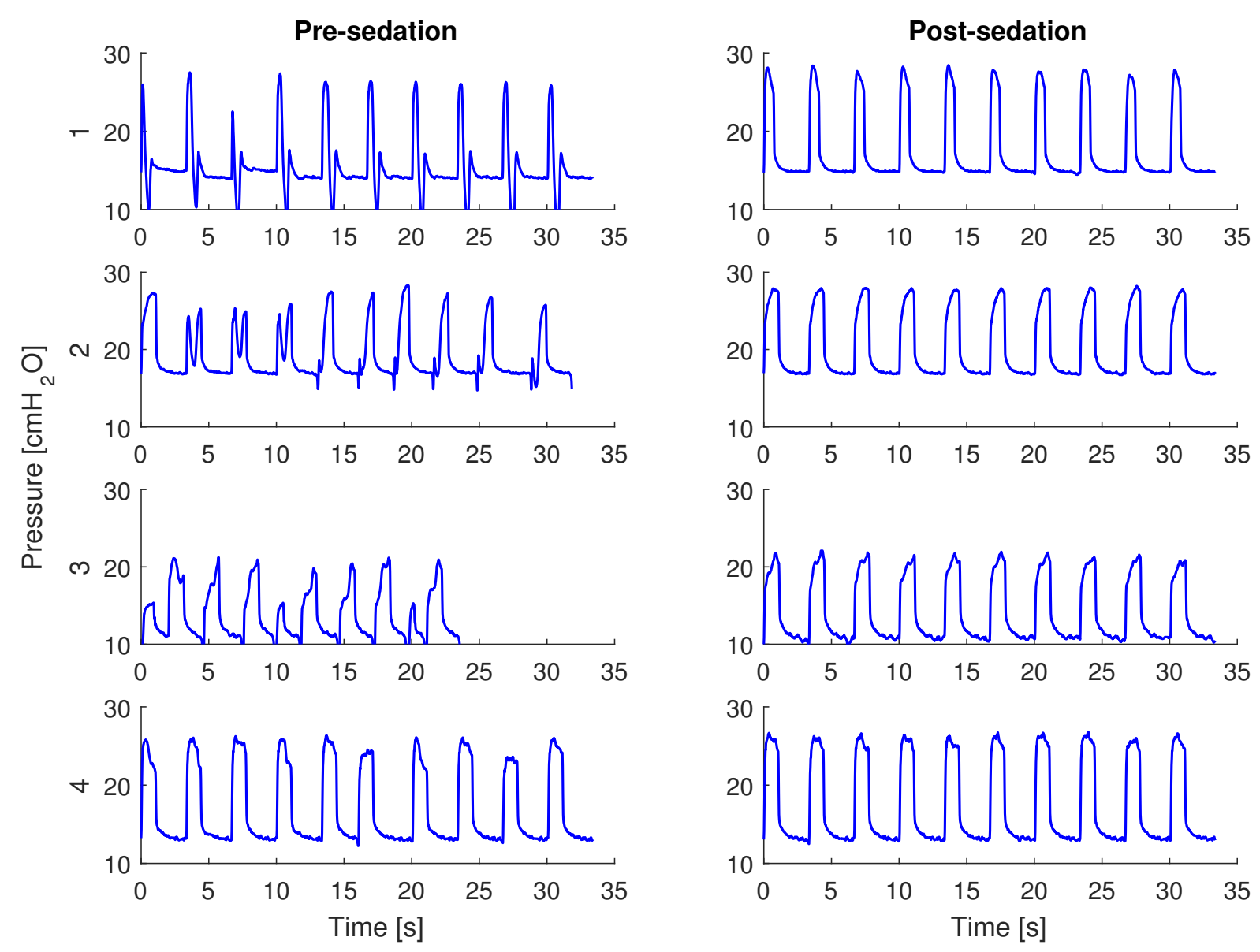

30


Figure 6: Pressure waveforms of the first 10 breathing cycles of each of the four data sets 
Dataset 2 with the polynomial model, and Dataset 3 with IIPR. Figure 8 shows that the smallest change in median estimated resistance in Dataset 1 is from the II Predator model, Datasets 2 and 4 with the single compartment model, and PREDATOR for Dataset 3. Additional numerical results are included in Appendix A, Tables A1 and A2 with a summary of the differences in distributions of estimated elastance and resistance.

The variability of the estimations of elastance and resistance are shown in Tables A3 and A4 present the median absolute deviations of the model identified parameters. Variability is also seen in Figures 7 and 8 where the cumulative distribution curves are less vertical.

The MAD elastance in the pre-sedation data is lowest for II PREDATOR in Datasets 1 and 4, while PREDATOR is lowest in Datasets 2 and 3. MAD resistance is lowest for II PREDATOR in Datasets 1,2 and 4, while PREDATOR has the most consistent resistance estimates in Dataset 3. Figure 9 shows a representation of performance of the models. It shows both the variability of the estimated elastance, and the change in median identified elastance after the administration of sedation. For each method, values closer to $(0,0)$ indicate better performance.

\section{Discussion}

\subsection{Single Compartment Model}

The single compartment model performs exceptionally poorly when there is any level of asynchronous patient effort. Figure 9 demonstrates how this model can have large differences in median identified elastance (+ symbols) in this baseline case, and also highly variable elastance and resistance estimations, as seen by the very high MAD for some patients in Table A3. This poor performance is expected as the model is passive and is not capable of capturing the effects of noisy and/or asynchronous breathing. The single compartment model is very simple, easy to identify with clinical data, and performs well when the patient is sedated with no spontaneous or asynchronous breathing, but is very sensitive to patient effort $[32,33]$. Figure 2a shows an example of overestimation of resistance and underestimation of elastance that occurs when the airway pressure in the middle of inspiration is greatly reduced due to patient effort. The single compartment model is included in this analysis as a baseline for comparison of performance against the other models, as it does not take into account patient effort. As such, the single compartment model is expected to have worse performance than the other models.

\subsection{Models to capture patient effort}

Both the constrained optimisation and the polynomial model are similar in the way they attempt to model the unknown patient effort that is acting on the basic single compartment model. By approximating the patient effort, it enables the respiratory mechanics representing the condition of the lung to be identified.

\subsubsection{Constrained optimisation}

The constrained optimisation approach, as presented by Vicario et al. [23], was designed for estimation of respiratory mechanics in spontaneously breathing patients during pressure control ventilation. In the comparison of methods presented here, the constrained optimisation approach has been applied to volume-control ventilation data with patient efforts that are more varied in timing and size than the data the model was developed with [23]. Because of the approach to fitting the model, it is limited to capturing patient efforts that occur in inspiration, though all other models presented here also only focus on inspiration.

The constrained optimisation model was not developed for identifying respiratory mechanics during asynchronous breathing. Instead, its goal was to capture the magnitude and timing of patient efforts. The modelled patient effort profile can then additionally be used to monitor the work of breathing [34]. In addition, it also captures respiratory mechanics, and thus offers a single method for both outcomes with no need for an extra comparison of pressure waveforms.

Figure 11a demonstrates constrained optimisation model performing well, on a breath where the patient effort is at the start, and in good synchrony with the ventilator effort. The constrained optimisation approach uses the entire breathing cycle of data, rather than just the inspiration part as used by the other models. As the length of time in the expiratory phase is relatively longer than inspiration, there is more data points, so the model is preferentially fitted to the expiration data. Respiratory mechanics seen in inspiration can vary from those identified during expiration [35, 36]. The preferential fitting of expiration can often result in poor model fitting in the region of inspiration unaffected by patient 

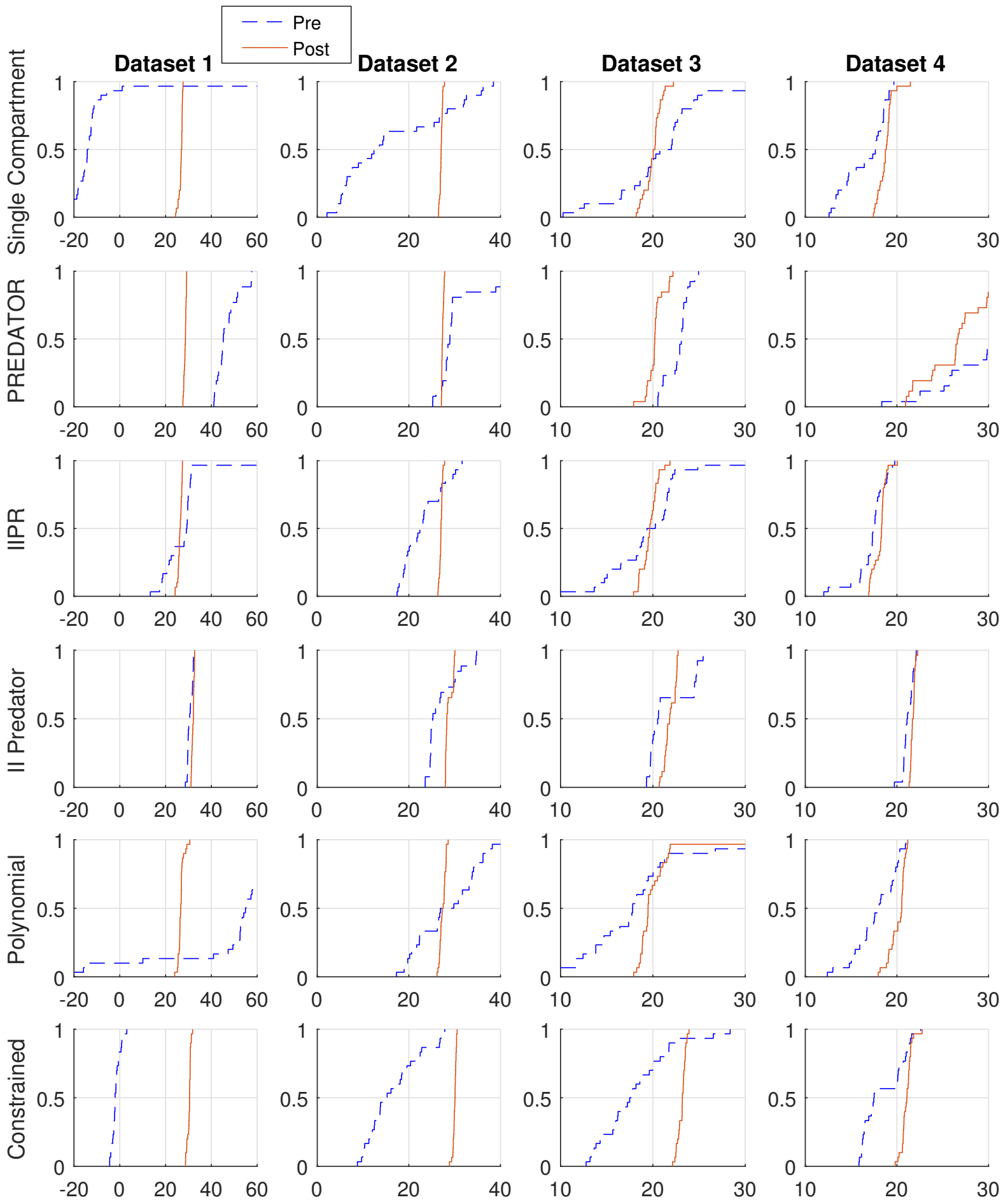

Elastance $\left[\mathrm{cmH}_{2} \mathrm{O} / \mathrm{L}\right]$

Figure 7: Distributions of elastance, $E$, $\left[\mathrm{cmH}_{2} \mathrm{O} / \mathrm{L}\right]$ across models and patients, showing both pre-sedation (dashed) and post-sedation (solid) breaths 

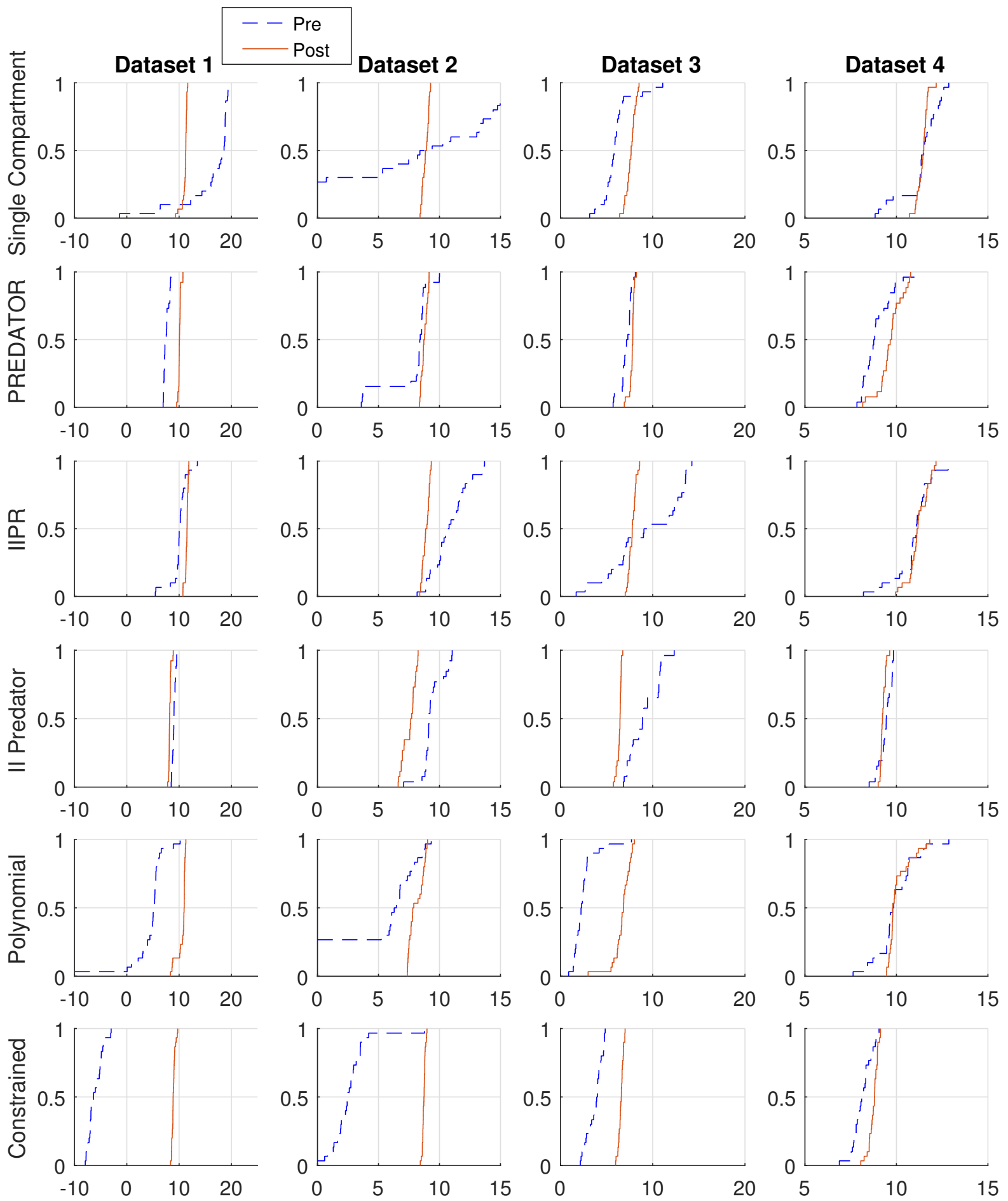

Resistance $\left[\mathrm{cmH}_{2} \mathrm{Os} / \mathrm{L}\right]$

Figure 8: Distributions of resistance, $R,\left[\mathrm{cmH}_{2} \mathrm{Os} / \mathrm{L}\right]$ across models and patients, showing both pre-sedation (dashed) and post-sedation (solid) breaths 


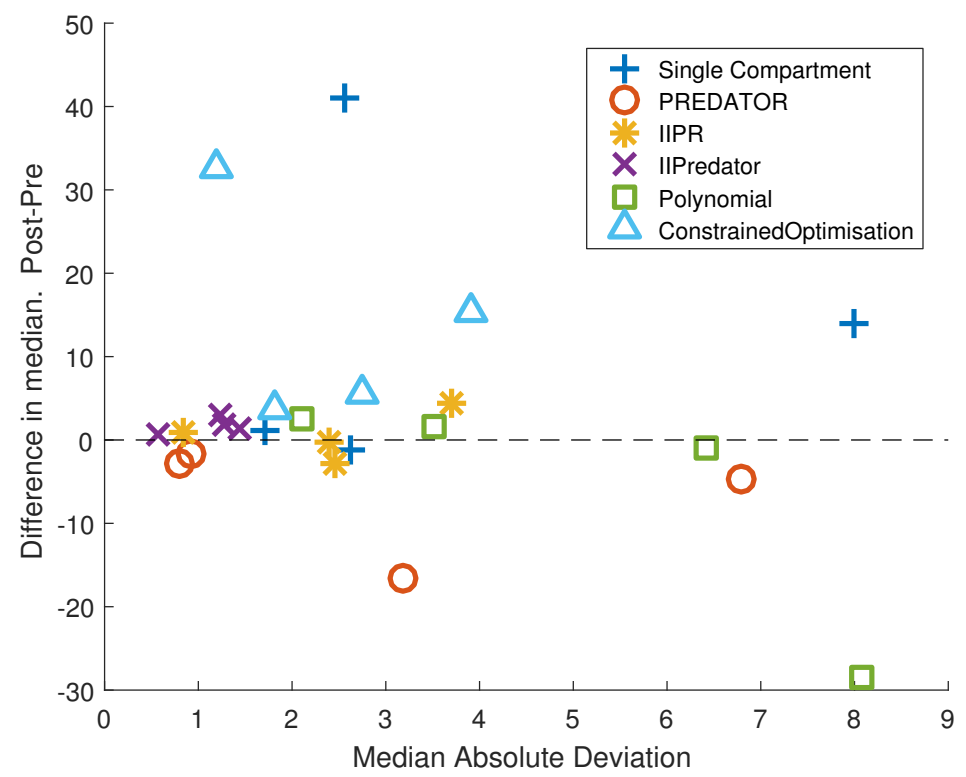

Figure 9: Graphical representation of performance of each model for elastance estimation for each Dataset. Vertical axis is the difference in medians of identified elastance for each model, pre- and post-sedation, with one marker for each patient. The horizontal axis is the sum of median absolute deviation (MAD) pre-sedation and post-sedation for each model and patient. Difference in medians, and MAD closer to 0 indicates superior performance of the model.

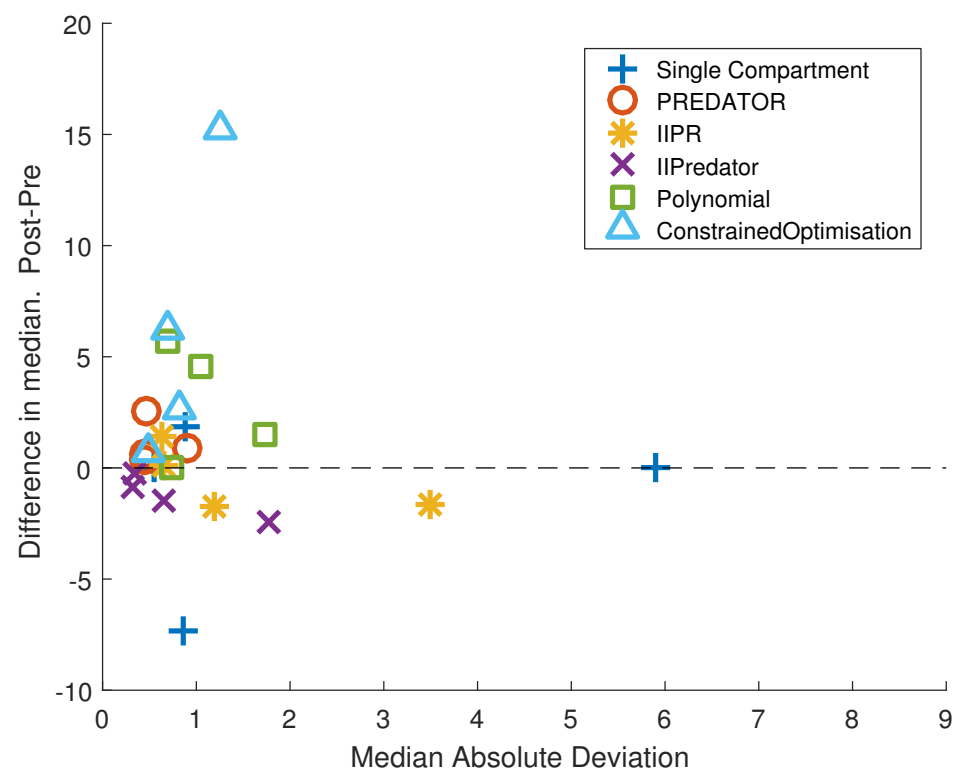

Figure 10: Performance of each model in estimating resistance for each Dataset. The PREDATOR model has the best estimates of resistance as it has a small difference in median estimated resistance after sedation, and a low median absolute deviation over all Datasets. 

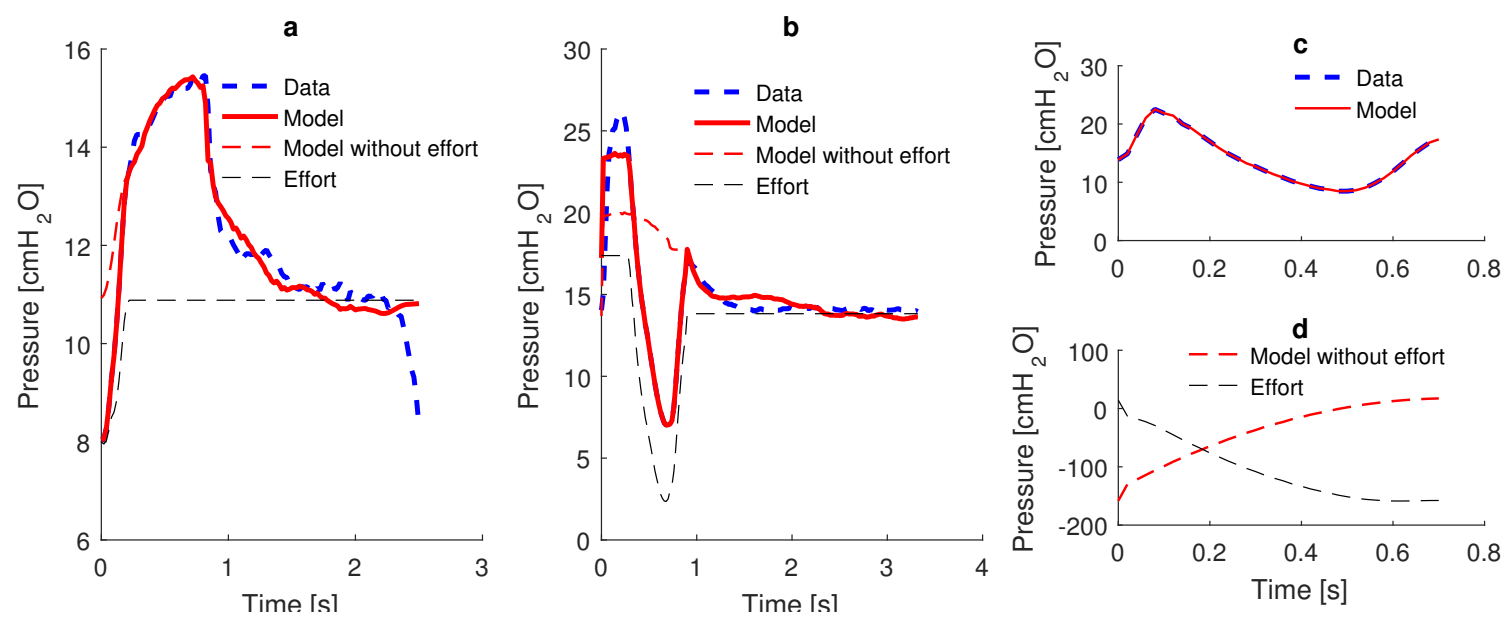

Figure 11: The constrained optimisation model. Part a shows where this model does not perform very well. The part of inspiration before the obvious patient effort has a very poor model fit, and subsequently poor estimation of elastance and resistance. Part $\mathbf{b}$ shows the model performing better, and capturing the position of patient effort used to trigger the breath. This breathing cycle is similar to the type of data that the model was developed on. Part $\mathbf{c}$ shows the same breathing cycle as part a, but with the model only fitted to inspiration, and the patient effort in $\mathbf{d}$. The identified parameters are physiologically implausible, $E \approx 400 \mathrm{cmH}_{2} \mathrm{O} / \mathrm{L}$ and $R \approx 0 \mathrm{cmH}_{2} \mathrm{Os} / \mathrm{L}$

effort. In general this is seen in the poor elastance estimates in the pre-sedation period in Figure 7. In particular, Figure 11b shows an example of the poor fitting in the early part of the breath.

To force preferential fitting of the data to the inspiration part of the cycle, the expiration part can be completely excluded. However, this choice presents further difficulties. The constraints applied to the patient effort profile are not sufficient to ensure a physiologically plausible effort profile, and the result can be a perfect fitting of the data in inspiration due to an unreasonably strong patient effort resulting in physiologically implausible estimations for $E$ and $R$. An example of this issue is seen in Figure 11c, where the constrained optimisation model has been fitted for inspiration only, to the same breath in part b. The lower panel shows the unreasonably large patient effort of $150 \mathrm{cmH}_{2} \mathrm{O}$ and the modelled pressure profile with patient effort removed. Finally, changing the weighting between expiration and inspiration yields results across this spectrum with similar issues.

Hence, the disadvantages of the model are problematic in this application. However, the model is likely to perform well in patient triggered spontaneous breathing modes [23, 34]. As a result, it performs better in cases for which it was designed, but less well in the highly variable Dataset used here, as seen in Figures 7 and 8.

\subsubsection{Polynomial model}

The polynomial model has reasonable success where the patient efforts occur for a small portion of the inspiration time. However, when the patient efforts are long, this model can give physiologically implausible results [21]. When the patient effort is long, there is less inspiratory pressure data unaffected by patient effort, and thus the elastance and resistance estimations become less reliable. This issue is evident in the highly variable results presented across all patients in Figure 7 for identified elastance before sedation.

The polynomial model is very similar to the constrained optimisation approach, though it has a far stricter constraint placed on the patient effort profile. Constrained optimisation only restricts the patient effort to be monotonically decreasing in the first part, followed by monotonically increasing, and then constant during expiration. Because the polynomial model places more constraints on the shape of the effort, it is less likely to fit the patient effort to the entire inspiration region, as can sometimes occur the constrained optimisation model. The main advantage of the polynomial model is the very simple patient effort profile, though this is also a limitation, as the exact shape of the unknown patient effort is specified in the model.

\subsection{Methods to mitigate the effects of patient effort}

The PREDATOR, IIPR, and the combination of these two methods, have been developed for the purpose of respiratory mechanics estimations in volume controlled ventilation when there is some form 
of disturbance to the pressure waveforms. Because of this design, model fitting error is not a good way to analyse the efficacy of these models. The models are not intended to closely fit to all of the measured data, as certain sections of the data are being rejected as asynchronous, or corrupted by the unmodelled input from the patient's spontaneous breathing efforts.

\subsubsection{PREDATOR}

The PREDATOR method has reasonably stable parameter estimations, as low MADs are seen in Datasets 2 and 3, with more variable estimates in Datasets 1 and 4 . Reasonably low variability is expected due to the pooling and stacking of breaths, though the stacking process also results in higher pressures in general than are seen in other models. The higher overall pressures come from using the maximum of each pressure as the best estimate of the waveform in the stacking and reconstruction process. These higher pressures result in a reasonably consistent higher estimation of elastance [27]. This offset is the main drawback of the PREDATOR method, and is apparent in Figure 9 where the PREDATOR model tends to have a higher elastance before sedation, due to the asynchronous breathing that occurs (only) then in this study. This outcome contrasts with the other methods which tend to have a slight increase in elastance after sedation is applied, which is consistent with a small amount of regional lung collapse due to added sedation [31]. PREDATOR has the advantage of being very conceptually simple in its implementation. The main limitation of this model is that it requires multiple breaths to obtain an estimate of respiratory mechanics. Thus, there is a lag in identified $E$ and $R$ when used in real-time. Additionally, consistent patient efforts very close to the start of inspiration leave a very small number of data points available for model identification which can lead to highly variable parameter estimations.

\subsection{2. $I I P R$}

IIPR is generally quite effective at identifying a stable estimate of respiratory mechanics in this data set. As can be seen in Figure 9 and Table A1, the change in median identified elastance is generally small, though the variability of the estimated elastance is larger than might be desired clinically. IIPR has an advantage over PREDATOR as it only requires one breath for reconstruction. The single breath advantage is only evident when using reconstruction during recruitment manoeuvres where breaths at each PEEP level may be limited. It thus maximises the data and breaths available in a way PREDATOR does not. A limitation of IIPR is that it is always a proxy and a guess for the correct shape while assuming that a passive lung should behave according to the single compartment model, as the assumption of linear single compartment behaviour is made as part of the reconstruction process.

\subsubsection{PREDATOR}

Combining IIPR and PREDATOR improved performance of the models presented here. In some Datasets, II PREDATOR has worse performance in variability of elastance estimate (Figure 7 and Table A3 Dataset 2 and 3) than PREDATOR, though in Dataset 1 and 4, PREDATOR has a highly variable elastance estimate. Figure 9 and Table A3 shows that II PREDATOR has the lowest mean MAD of elastance, and that II PREDATOR has the least variable elastance estimates across all patient data.

II PREDATOR does have more variable estimates of resistance overall than PREDATOR, as seen in Figure 10 and Table A4. To guide treatment, elastance is used more than resistance[24, 37, 38], so a consistent elastance estimate is more clinically beneficial than a consistent resistance estimate. II PREDATOR has the same disadvantage as PREDATOR as it requires five breaths to pool together to make a respiratory mechanics estimate, though it does have the best performance in elastance estimates of all the models tested.

\subsection{Model Comparison}

From the results, II PREDATOR has the best performance in general of all the models on the data tested. Figure 9 shows that in general, II PREDATOR has lower median absolute deviations, suggesting more consistent elastance estimation across the pre- and post-sedation range, and lower difference in medians. Figure 10 shows II PREDATOR is also reasonably good at resistance estimations, though has slightly worse performance than PREDATOR for resistance estimations.

It is hypothesised that the true respiratory system resistance should not vary too much when sedation is applied, as there should be very little change in underlying lung condition over such a short time with no change in MV settings. Respiratory system elastance could be expected to either stay the same or 
have a relatively small increase to account for any regional lung collapse. Thus, consistency over this range in Figures 7 and 8, and Tables A1 to A4, with values closer to (0,0) in Figures 9 and 10, indicates that II PREDATOR is a better method.

However, depending on the particular respiratory mechanics application, other models may be more suited to specific applications. For example, in long term monitoring of a patients condition, where the level of spontaneous breathing is changing over time, a small difference in medians is the most important, and a higher level of variation in parameter estimations may be tolerated. A smaller change in median is also desirable when comparing using respiratory mechanics in conjunction with a lung imaging technique. In this situation, having a "correct" value for respiratory mechanics during sedation, and when patients breathe spontaneously is most important.

For assessing respiratory mechanics over a short period of time, such as during a short staircase recruitment manoeuvre, where there may only a limited number of breaths at each PEEP level, a lower MAD is more important. During a recruitment manoeuvre, if sedation is kept constant, only the relative changes in mechanics during PEEP changes is of interest, and therefore less variability in the parameter estimations is desirable. If PEEP selection is being guided by a particular parameter, such as minimal elastance [37,39], then the most appropriate model is the one that provides the lowest MAD for the parameter of interest. Tables A3 and A4 show II PREDATOR has the lowest MAD elastance and PREDATOR has the lowest MAD resistance

\subsection{Data}

This study relies on having data recorded exactly when patients have sedation administered for clinical reasons. This type of data allows the analysis of models to determine how the models perform with and without patient effort for a very similar lung condition and unchanged MV settings. Hence, the observational data we have available is somewhat limited and not consistent. While the analysis would be improved with more data, the varied nature of the data that is presented in this work (see Figure 6) means the results are probably reliable. Further validation of the results presented here could be achieved with a larger observational or interventional trial.

A true validation of the respiratory mechanics during spontaneous breathing would only be available with a measure of pleural pressure, as obtained from an oesophageal pressure catheter. In the absence of this additional invasive monitoring, data from the same patient before and after sedation, provides a reasonable validation of performance of the models during spontaneous breathing effort.

It is beneficial to have analysis performed with noisy data from clinical observation rather than the "clean" and "useful" data that is often used to develop models. Adding gaussian noise to simulated breathing cycles may be beneficial in testing the practical identifiability of the respiratory mechanics model. However, in a clinical scenario, the variability in patient effort tends to be highly inconsistent [40], and for models to be clinically applicable, they must be robust to the broad range of data. Hence, analysis using the type of data presented here is useful and clinically important.

\subsection{Limitations of the study}

A comparison of model fitting error is not performed here. This is because a lower model fitting error is guaranteed in some models because of the process of reconstruction, or having many more free parameters. In addition, fitting the recorded data very closely does not necessarily mean the model is good. Specifically, there are regions in many breaths where the data is not expected to match the model due to the patient effort. Hence model fitting error is not a suitable parameter in this study.

This work solely uses data from volume control ventilation. Therefore, the suggestion of the best model and method to use in the presence of variable patient effort is limited to this case. Some of these models may also work in pressure control ventilation modes, but this would require a separate study or analysis for which data was unavailable.

\section{Conclusions}

In this study, several existing methods for estimating respiratory mechanics during spontaneous and asynchronous breathing were evaluated. Each model presents its own unique advantages but equally have their own application limitations. We have found that extending the IIPR with PREDATOR has improved performance compared to previous methods. In this unique Dataset analysis, II PREDATOR is the preferred method for respiratory mechanics estimation in volume control ventilation modes where there is variable added patient-specific spontaneous and asynchronous breathing effort. This method 
provides a relatively stable estimate of respiratory mechanics with small changes in identified parameters when sedation is applied and the patient effort ceases. Hence, it could be effectively used to extend model-based methods for guiding MV to the relatively frequent cases where some patient spontaneous breathing efforts are evident.

\section{Acknowledgements}

This work was partially supported by HRC Grant 13/213, EU FP7/RSNZ Marie Curie IRSES Grant and NZ MedTech CoRE funding.

\section{References}

[1] Ashwath Sundaresan and J. Geoffrey Chase. Positive end expiratory pressure in patients with acute respiratory distress syndrome The past, present and future. Biomed. Signal Process. Control, 7(2):93-103, mar 2012.

[2] Fleur T Tehrani and James H Roum. Intelligent decision support systems for mechanical ventilation. Artif. Intell. Med., 44(3):171-82, nov 2008.

[3] Anup Das, Prathyush P Menon, Jonathan G Hardman, and Declan G Bates. Optimization of Mechanical Ventilator Settings for Pulmonary Disease States. Biomed. Eng. IEEE Trans., 60(6):1599-1607, 2013.

[4] Zhanqi Zhao, Marcus Eger, Thomas Handzsuj, V Marco Ranieri, Lorenzo Appendini, and Cosimo Micelli. Noninvasive Method for Measuring Respiratory System Compliance during Pressure Support Ventilation. In Annu. Int. Conf. IEEE EMBS, volume 33, pages 3808-3811, Boston, 2011.

[5] Kristel Lopez-Navas, Sebastian Brandt, Merle Strutz, Hartmut Gehring, and Ullrich Wenkebach. Non-invasive determination of respiratory effort in spontaneous breathing and support ventilation: a validation study with healthy volunteers. Biomed. Eng. / Biomed. Tech., 59(4), 2014.

[6] Nor Salwa Damanhuri, Yeong Shiong Chiew, Nor Azlan Othman, Paul D. Docherty, Christopher G. Pretty, Geoffrey M. Shaw, Thomas Desaive, and J. Geoffrey Chase. Assessing Respiratory Mechanics Using Pressure Reconstruction Method in Mechanically Ventilated Spontaneous Breathing Patient. Comput. Methods Programs Biomed., 2016.

[7] Catherine S. H. Sassoon and Guy T. Foster. Patient-ventilator asynchrony. Curr. Opin. Crit. Care, 7(1):28-33, feb 2001.

[8] Scott K Epstein. How often does patient-ventilator asynchrony occur and what are the consequences? Respir. Care, 56(1):25-38, jan 2011

[9] Evangelia Akoumianaki, Aissam Lyazidi, Nathalie Rey, Dimitrios Matamis, Nelly Perez-Martinez, Raphael Giraud, Jordi Mancebo, Laurent Brochard, and Jean-Christophe Marie Richard. Mechanical ventilation-induced reversetriggered breaths: a frequently unrecognized form of neuromechanical coupling. Chest, 143(4):927-38, apr 2013.

[10] Jason H.T. Bates. Lung mechanics: an inverse modeling approach. Cambridge University Press, New York, 2009.

[11] Christoph Schranz, Paul D Docherty, Yeong Shiong Chiew, J Geoffrey Chase, and Knut Möller. Structural identifiability and practical applicability of an alveolar recruitment model for ARDS patients. IEEE Trans. Biomed. Eng., 59(12):3396-404, dec 2012.

[12] Christoph Schranz, Paul D Docherty, Yeong Shiong Chiew, Knut Möller, and J Geoffrey Chase. Identifiability Analysis of a Pressure-Depending Alveolar Recruitment Model. In IFAC Symp. Biol. Med. Syst., volume 8, pages 137-142, 2012.

[13] G Polese, A Serra, and A Rossi. Respiratory mechanics in the intensive care unit. In R. Gosselink and H. Stam, editors, Lung Funct. Test., chapter 10, pages 195-206. European Respiratory Society, 2005.

[14] Sonia Khirani, Guido Polese, Andrea Aliverti, Lorenzo Appendini, Gianluca Nucci, Antonio Pedotti, Michele Colledan, Alessandro Lucianetti, Pierre Baconnier, and Andrea Rossi. On-line monitoring of lung mechanics during spontaneous breathing: a physiological study. Respir. Med., 104(3):463-71, mar 2010.

[15] Joshua O Benditt. Esophageal and gastric pressure measurements. Respir. Care, 50(1):68-75; discussion 75-77, 2005.

[16] D Navajas and R Farré. Forced oscillation assessment of respiratory mechanics in ventilated patients. Crit. Care, $5(1): 3-9,2001$.

[17] E Oostveen, D MacLeod, H Lorino, R Farré, Z Hantos, K Desager, and F Marchal. The forced oscillation technique in clinical practice: methodology, recommendations and future developments. Eur. Respir. J. Off. J. Eur. Soc. Clin. Respir. Physiol., 22:1026-1041, 2003.

[18] Peter Kostic, Emanuela Zannin, Marie Andersson Olerud, Pasquale P Pompilio, Göran Hedenstierna, Antonio Pedotti, Anders Larsson, Peter Frykholm, and Raffaele L Dellaca. Positive end-expiratory pressure optimization with forced oscillation technique reduces ventilator induced lung injury: a controlled experimental study in pigs with saline lavage lung injury. Crit. Care, 15(3):R126, 2011.

[19] Arnaud W Thille, Pablo Rodriguez, Belen Cabello, François Lellouche, and Laurent Brochard. Patient-ventilator asynchrony during assisted mechanical ventilation. Intensive Care Med., 32(10):1515-22, oct 2006.

[20] Vincent Major, Simon Corbett, Daniel Redmond, Alex Beatson, Daniel Glassenbury, Yeong Shiong Chiew, Christopher Pretty, Thomas Desaive, Ákos Szlávecz, Balázs Benyó, Geoffrey M. Shaw, and J. Geoffrey Chase. Respiratory mechanics assessment for reverse-triggered breathing cycles using pressure reconstruction. Biomed. Signal Process. Control, 23:1-9, 2016.

[21] Daniel P Redmond, Paul D Docherty, Yeong Shiong Chiew, and J Geoffrey Chase. A Polynomial Model of Patientspecific Breathing Effort During Controlled Mechanical Ventilation. In Annu. Int. Conf. IEEE EMBS, volume 37, pages 4532-4535, Milan, 2015.

[22] N Damanhuri, Y. S. Chiew, N. A. Othman, P. D. Docherty, G. M. Shaw, and J. G. Chase. Pressure reconstruction method for spontaneous breathing effort monitoring. Crit. Care, 19(Suppl 1):S90-91, 2015. 
[23] Francesco Vicario, Antonio Albanese, Nikolaos Karamolegkos, Dong Wang, Adam Seiver, and Nicolas Chbat. Noninvasive Estimation of Respiratory Mechanics in Spontaneously Breathing Ventilated Patients: A Constrained Optimization Approach. IEEE Trans. Biomed. Eng., 0018(c):1-1, 2015.

[24] Yeong Shiong Chiew, J Geoffrey Chase, Geoffrey M Shaw, Ashwath Sundaresan, and Thomas Desaive. Model-based PEEP optimisation in mechanical ventilation. Biomed. Eng. Online, 10(1):111, jan 2011.

[25] Erwin J van Drunen, J Geoffrey Chase, Yeong Shiong Chiew, Geoffrey M Shaw, and Thomas Desaive. Analysis of different model-based approaches for estimating dFRC for real-time application. Biomed. Eng. Online, 12(1):9, 2013.

[26] Laurent Brochard, Greg S Martin, Lluis Blanch, Paolo Pelosi, F Javier Belda, Amal Jubran, Luciano Gattinoni, Jordi Mancebo, V Marco Ranieri, Jean-Christophe M Richard, Diederik Gommers, Antoine Vieillard-Baron, Antonio Pesenti, Samir Jaber, Ola Stenqvist, and Jean-Louis Vincent. Clinical review: Respiratory monitoring in the ICU - a consensus of 16. Crit. Care, 16(2):219, jan 2012.

[27] Daniel P Redmond, Vincent Major, Simon Corbett, Daniel Glassenbury, Alex Beatson, Yeong Shiong Chiew, Geoffrey M Shaw, and J Geoffrey Chase. Pressure reconstruction by eliminating the demand effect of spontaneous respiration ( PREDATOR ) method for assessing respiratory mechanics of reverse-triggered breathing cycles. In $I E E E$ Conf. Biomed. Eng. Sci., volume 1, pages 332-337, Kuala Lumpur, 2014.

[28] Vincent Major, Simon Corbett, Daniel Redmond, Alex Beatson, Daniel Glassenbury, Yeong Shiong Chiew, Christopher Pretty, Thomas Desaive, Akos Szlavecz, Balazs Benyo, Geoffrey M Shaw, and J Geoffrey Chase. Assessing Respiratory Mechanics of Reverse-Triggered Breathing Cycles Case Study of Two Mechanically Ventilated Patients. In IFAC Symp. Biol. Med. Syst., volume 9, Berlin, 2015.

[29] Felix Newberry, D Oliver Kannangara, Sarah Howe, Vincent Major, Daniel Redmond, Akos Szlavecz, Yeong Shiong Chiew, Balazs Benyo, Geoffrey M Shaw, and J Geoffrey Chase. Iterative Interpolative Pressure Reconstruction for Improved Respiratory Mechanics Estimation during Asynchronous Volume Controlled Ventilation. In Int. Conf. Innov. Biomed. Eng. Life Sci., pages 1-6, Putrajaya, Malaysia, 2015.

[30] Kyeong T. Kim, Yeong Shiong Chiew, Christopher Pretty, Geoffrey M. Shaw, Thomas Desaive, and J. Geoffrey Chase. Breath-to-breath respiratory mechanics variation: how much variation should we expect. Crit. Care, 19(Suppl 1):S91, 2015.

[31] J C Richard, S M Maggiore, B Jonson, J Mancebo, F Lemaire, and L Brochard. Influence of tidal volume on alveolar recruitment. Respective role of PEEP and a recruitment maneuver. Am. J. Respir. Crit. Care Med., 163(7):1609-13, jun 2001.

[32] Yeong Shiong Chiew, Sarah F Poole, Daniel P Redmond, Erwin J van Drunen, Nor Salwa Damanhuri, Christopher Pretty, Paul D Docherty, Bernard Lambermont, Geoffrey M Shaw, Thomas Desaive, and J Geoffrey Chase. TimeVarying Respiratory Elastance for Spontaneously Breathing Patients. In IFAC World Congr., volume 19, Cape Town, 2014.

[33] Daniel Gilstrap and Neil MacIntyre. Patient-ventilator interactions. Implications for clinical management. Am. J. Respir. Crit. Care Med., 188(9):1058-68, nov 2013.

[34] Francesco Vicario, Antonio Albanese, Dong Wang, Nikolaos Karamolegkos, and Nicolas W Chbat. Constrained Optimization for Noninvasive Estimation of Work of Breathing. In Annu. Int. Conf. IEEE EMBS, volume 37, pages 5327-5330, Milan, 2015.

[35] Erwin J van Drunen, Yeong Shiong Chiew, J Geoffrey Chase, Geoffrey M Shaw, Bernard Lambermont, Nathalie Janssen, Nor Salwa Damanhuri, and Thomas Desaive. Expiratory model-based method to monitor ARDS disease state. Biomed. Eng. Online, 12(1):57, jan 2013.

[36] Daniel P. Redmond, Jörn Kretschmer, Yeong Shiong Chiew, Christopher Pretty, Knut Möller, and J. Geoffrey Chase. Modelling Expiratory using Viscoelastic Pressure Dependant Recruitment Models - Is it the same as inspiration. In Congr. Int. Soc. Biomech., volume 25, pages 88-89, Glasgow, 2015.

[37] Alysson Roncally S Carvalho, Frederico C Jandre, Alexandre V Pino, Fernando a Bozza, Jorge Salluh, Rosana Rodrigues, Fabio O Ascoli, and Antonio Giannella-Neto. Positive end-expiratory pressure at minimal respiratory elastance represents the best compromise between mechanical stress and lung aeration in oleic acid induced lung injury. Crit. Care, 11(4):R86, jan 2007.

[38] Fernando Suarez-Sipmann, Stephan H Böhm, Gerardo Tusman, Tanja Pesch, Oliver Thamm, Hajo Reissmann, Andreas Reske, Anders Magnusson, and Göran Hedenstierna. Use of dynamic compliance for open lung positive endexpiratory pressure titration in an experimental study. Crit. Care Med., 35(1):214-21, jan 2007.

[39] Yeong Shiong Chiew, Christopher G Pretty, Geoffrey M Shaw, Yeong Woei Chiew, Bernard Lambermont, Thomas Desaive, and J Geoffrey Chase. Feasibility of titrating PEEP to minimum elastance for mechanically ventilated patients. Pilot Feasibility Stud., 1:1-10, 2015.

[40] Jennifer L. Dickson, Cameron A. Gunn, and J. Geoffrey Chase. Humans are Horribly Variable. Int. J. Clin. Med. Imaging, 1(2):1000142, 2014.

\section{Appendix A. Additional Results}


Table A1: Median [Interquartile range] of $E\left[\mathrm{cmH}_{2} \mathrm{O} / \mathrm{L}\right]$ pre- and post-sedation for each patient identified with each of the six different models. $\Delta$ is the difference in median, lower quartile and upper quartile of identified $E$ after sedation is applied

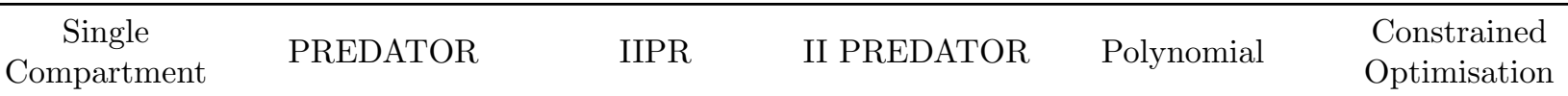

Pre -14.0[-17.6: -12.0] $45.2[43.1: 48.9] \quad 29.1[20.5: 30] \quad 30.4[29.7: 31.8] \quad 54.5[49.4: 64.1] \quad-1.70[-2.49:-0.54]$ 1 Post $27.0[26.5: 27.2] \quad 28.7[28.1: 28.9] \quad 26.1[25.6: 26.8] 32.1[31.5: 32.4] \quad 26.4[26.1: 27] \quad 30.5[29.7: 30.8]$ $\Delta \quad 41.0[44.1: 39.2] \quad-16.6[-15:-19.9] \quad-3.01[5.08:-3.27] 1.74[1.78-0.59] \quad-28.1[-23.3:-37.1] 32.2[32.2: 31.3]$

Pre 45.2[43.1:48.9] $29.1[20.5: 30] \quad 54.5[49.4: 64.1] 25.2[24.8: 29.8] \quad-1.7[-2.49:-0.54] 30.4[29.7: 31.8]$ 2 Post $28.7[28.1: 28.9] \quad 26.1[25.6: 26.8] \quad 26.4[26.1: 27] \quad 28.3[28.0: 29.8] \quad 30.5[29.7: 30.8] \quad 32.1[31.5: 32.4]$ $\Delta \quad 14.1[20.5:-0.74]-1.62[-0.97:-1.94] 4.55[7.7: 0.57] \quad 3.09[3.27:-0.09]-1.1[5.11:-5.8] \quad 13.2[17.3: 8.91]$

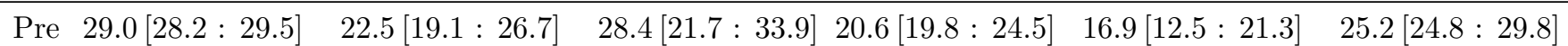
3 Post $27.3[27.2: 27.5] \quad 27.1[26.8: 27.2] \quad 27.3[26.8: 28.1] 21.8[21.4: 22.5] \quad 30.0[29.8: 30.2] \quad 28.3[28: 29.8]$ $\Delta-1.25[0.92:-2.31]-2.87[-2.59:-2.92] 0.64[2.95:-1.23] 1.26[1.60:-2.02] \quad 1.7[5.17: 0.82] \quad 5.87[8.23: 3.49]$

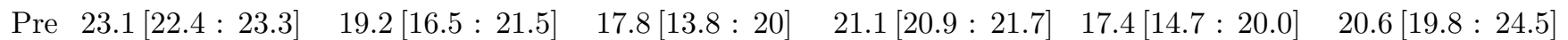
4 Post $20.2[19.8: 20.4] \quad 19.8[19.5: 20.3] \quad 19.5[19.0: 20.8] 21.7[21.6: 21.9] \quad 23.2[22.9: 23.5] \quad 21.8[21.4: 22.5]$

$\Delta \quad 1.14[3.64: 0.60] \quad-4.7[-2.11:-3.44] \quad 1.0[1.64: 0.76] \quad 0.62[0.70: 0.24] \quad 2.5[3.12: 1.09] \quad 3.65[4.37: 1.2]$

Table A2: Median [IQR] of $R\left[\mathrm{cmH}_{2} \mathrm{Os} / \mathrm{L}\right]$ pre- and post-sedation for each patient identified with each of the six different models. $\Delta$ is the difference in median, lower quartile and upper quartile of identified $R$ after sedation is applied

\begin{tabular}{cccccccc}
\hline & $\begin{array}{c}\text { Single } \\
\text { Compartment }\end{array}$ & PREDATOR & IIPR & II PREDATOR & Polynomial & $\begin{array}{c}\text { Constrained } \\
\text { Optimisation }\end{array}$ \\
\hline Pre & $18.6[16.1: 18.8]$ & $7.48[7.12: 8.03]$ & $10.2[9.72: 10.8]$ & $9.01[8.81: 9.22]$ & $5.3[4.41: 5.62]$ & $-5.94[-7.0:-4.89]$ \\
$\mathbf{1}$ Post & $11.3[11.1: 11.4]$ & $10.1[9.99: 10.2]$ & $11.5[11.3: 11.7]$ & $8.19[8.11: 8.31]$ & $11[10.3: 11.1]$ & $8.91[8.69: 9.11]$ \\
$\Delta$ & $-7.3[-5.0:-7.44]$ & $2.58[2.86: 2.18]$ & $1.32[1.59: 0.88]$ & $-0.82[-0.70:-0.91] 5.68[5.85: 5.46]$ & $14.8[15.7: 14.0]$ \\
\hline Pre & $8.90[-0.89: 14.2]$ & $8.4[8.16: 8.62]$ & $10.7[10.1: 11.9]$ & $9.14[8.96: 9.59]$ & $6.06[-4.09: 7.37]$ & $2.48[1.89: 2.96]$ \\
$\mathbf{2}$ Post & $8.90[8.62: 9.12]$ & $8.75[8.56: 8.97]$ & $8.84[8.59: 9.1]$ & $7.68[6.96: 8.00]$ & $8.07[7.5: 8.69]$ & $8.7[8.64: 8.79]$ \\
$\Delta$ & $-0.002[9.51:-5.08]$ & $0.34[0.40: 0.35]-1.81[-1.51:-2.81]$ & $-1.46[-2.0:-1.59]$ & $2.0[11.6: 1.32]$ & $6.21[6.75: 5.83]$ \\
\hline Pre & $5.82[5.16: 6.31]$ & $7.23[6.77: 7.57]$ & $9.45[6.78: 13.2]$ & $8.90[7.57: 10.7]$ & $2.34[1.79: 2.76]$ & $3.97[3.25: 4.42]$ \\
$\mathbf{3}$ Post & $7.66[7.30: 7.92]$ & $7.84[7.65: 7.9]$ & $7.78[7.47: 8.03]$ & $6.46[6.31: 6.55]$ & $6.76[6.23: 7.2]$ & $6.53[6.34: 6.71]$ \\
$\Delta$ & $1.85[2.13: 1.61]$ & $0.61[0.88: 0.33]-1.68[0.69:-5.12]$ & $-2.44[-1.27:-4.12] 4.42[4.43: 4.43]$ & $2.56[3.1: 2.29]$ \\
\hline Pre & $11.5[11.3: 12]$ & $8.79[8.45: 9.53]$ & $11.1[10.8: 11.5]$ & $9.45[9.28: 9.76]$ & $9.82[9.47: 10.6]$ & $8.09[7.78: 8.34]$ \\
$\mathbf{4}$ Post & $11.5[11.3: 11.6]$ & $9.7[9.28: 9.99]$ & $11.2[10.8: 11.7]$ & $9.24[9.17: 9.4]$ & $9.89[9.76:$ & $10.5]$ & $8.8[8.62: 8.93]$ \\
$\Delta$ & $-0.01[-0.01:-0.42]$ & $0.91[0.84: 0.46]$ & $0.07[0.03: 0.16]$ & $-0.21[-0.11:-0.36]$ & $0.07[0.30:-0.10]$ & $0.71[0.84: 0.60]$ \\
\hline
\end{tabular}

Table A3: Median absolute deviations of identified elastance, $E\left[\mathrm{cmH}_{2} \mathrm{O} / \mathrm{L}\right]$, for each model in each Dataset.

\begin{tabular}{|c|c|c|c|c|c|c|c|}
\hline & & $\begin{array}{c}\text { Single } \\
\text { Compartment }\end{array}$ & PREDATOR & IIPR & II PREDATOR & Polynomial & $\begin{array}{l}\text { Constrained } \\
\text { Optimisation }\end{array}$ \\
\hline \multirow{2}{*}{1} & Pre & 2.21 & 2.85 & 2.02 & 0.818 & 7.65 & 1.12 \\
\hline & Post & 0.347 & 0.335 & 0.591 & 0.455 & 0.455 & 0.328 \\
\hline \multirow{2}{*}{2} & Pre & 7.80 & 0.765 & 3.50 & 0.946 & 6.35 & 4.43 \\
\hline & Post & 0.19 & 0.155 & 0.161 & 0.284 & 0.529 & 0.222 \\
\hline \multirow{2}{*}{3} & Pre & 2.12 & 0.536 & 2.49 & 0.863 & 3.20 & 2.67 \\
\hline & Post & 0.509 & 0.267 & 0.443 & 0.584 & 0.642 & 0.265 \\
\hline \multirow{2}{*}{4} & Pre & 1.35 & 4.02 & 0.506 & 0.392 & 1.64 & 1.44 \\
\hline & Post & 0.356 & 2.78 & 0.257 & 0.185 & 0.413 & 0.381 \\
\hline & Mean & 1.86 & 1.46 & 1.25 & 0.566 & 2.61 & 1.36 \\
\hline
\end{tabular}


Table A4: Median absolute deviations of identified resistance, $R\left[\mathrm{cmH}_{2} \mathrm{Os} / \mathrm{L}\right]$, for each model in each Dataset.

\begin{tabular}{|c|c|c|c|c|c|c|c|}
\hline & & $\begin{array}{c}\text { Single } \\
\text { Compartment }\end{array}$ & PREDATOR & IIPR & II PREDATOR & Polynomial & $\begin{array}{l}\text { Constrained } \\
\text { Optimisation }\end{array}$ \\
\hline \multirow{2}{*}{1} & Pre & 0.712 & 0.364 & 0.554 & 0.211 & 0.571 & 1.05 \\
\hline & Post & 0.144 & 0.108 & 0.181 & 0.118 & 0.162 & 0.215 \\
\hline \multirow{2}{*}{2} & Pre & 5.68 & 0.229 & 0.922 & 0.231 & 1.59 & 0.580 \\
\hline & Post & 0.223 & 0.215 & 0.257 & 0.419 & 0.588 & 0.0711 \\
\hline \multirow{2}{*}{3} & Pre & 0.553 & 0.349 & 3.20 & 1.67 & 0.469 & 0.705 \\
\hline & Post & 0.331 & 0.0937 & 0.280 & 0.112 & 0.488 & 0.184 \\
\hline \multirow{2}{*}{4} & Pre & 0.386 & 0.557 & 0.375 & 0.255 & 0.594 & 0.305 \\
\hline & Post & 0.154 & 0.354 & 0.400 & 0.0805 & 0.232 & 0.157 \\
\hline \multicolumn{2}{|r|}{ Mean } & 1.02 & 0.284 & 0.771 & 0.387 & 0.587 & 0.408 \\
\hline
\end{tabular}

